\title{
Fully Automated Screening of Immunocytochemically Stained Specimens for Early Cancer Detection
}

\author{
André A. Bell and Timna E. Schneider and Dirk A. C. Müller-Frank and \\ Dietrich Meyer-Ebrecht and Alfred Böcking and Til Aach \\ Institute of Imaging and Computer Vision \\ RWTH Aachen University, 52056 Aachen, Germany \\ tel: +49 24180 27860, fax: +49 2418022200 \\ web: www.Ifb.rwth-aachen.de
}

in: SPIE Medical Imaging 2007, Computer-Aided Diagnosis. See also BIBTEX entry below.

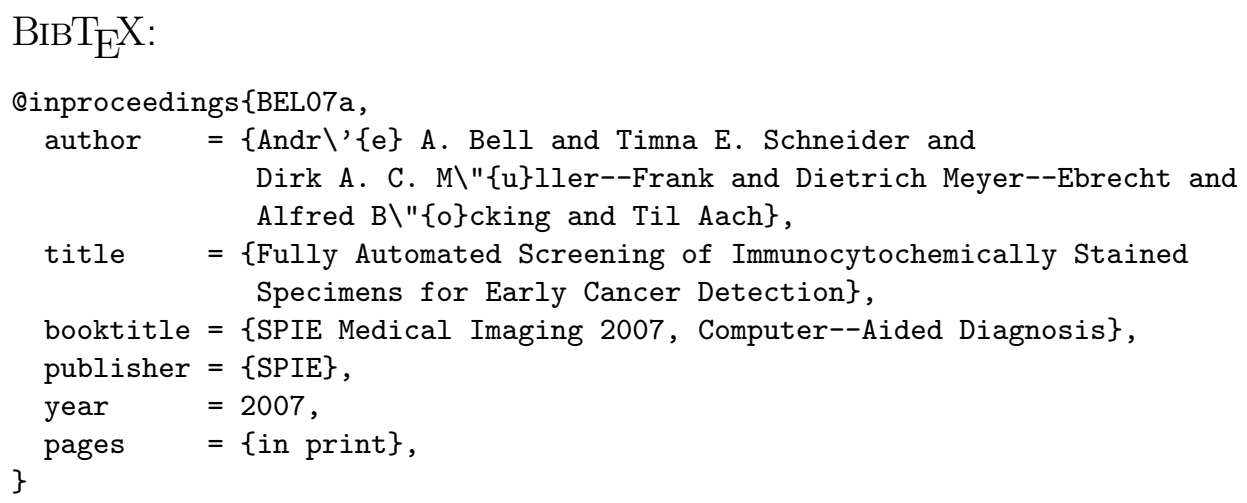

(C) 2007 Society of Photo-Optical Instrumentation Engineers. This paper was published in SPIE Medical Imaging 2007, Computer-Aided Diagnosis and is made available as an electronic reprint with permission of SPIE. One print or electronic copy may be made for personal use only. Systematic or multiple reproduction, distribution to multiple locations via electronic or other means, duplication of any material in this paper for a fee or for commercial purposes, or modification of the content of the paper are prohibited. 



\title{
Fully Automated Screening of Immunocytochemically Stained Specimens for Early Cancer Detection
}

\author{
André A. Bell ${ }^{a}$, Timna E. Schneider ${ }^{a}$, Dirk A. C. Müller-Frank ${ }^{a}$, \\ Dietrich Meyer-Ebrecht ${ }^{a}$, Alfred Böcking ${ }^{b}$ and Til Aach ${ }^{a}$ \\ ${ }^{a}$ Institute of Imaging and Computer Vision, RWTH Aachen University \\ Templergraben 55, 52056 Aachen, Germany \\ ${ }^{b}$ Institute of Cytopathology, Heinrich-Heine-University Düsseldorf \\ Moorenstr. 5, 40225 Düsseldorf, Germany
}

\begin{abstract}
Cytopathological cancer diagnoses can be obtained less invasive than histopathological investigations. Cells containing specimens can be obtained without pain or discomfort, bloody biopsies are avoided, and the diagnosis can, in some cases, even be made earlier. Since no tissue biopsies are necessary these methods can also be used in screening applications, e.g., for cervical cancer. Among the cytopathological methods a diagnosis based on the analysis of the amount of DNA in individual cells achieves high sensitivity and specificity. Yet this analysis is time consuming, which is prohibitive for a screening application. Hence, it will be advantageous to retain, by a preceding selection step, only a subset of suspicious specimens. This can be achieved using highly sensitive immunocytochemical markers like p16 ${ }^{i n k} 4 a$ for preselection of suspicious cells and specimens.

We present a method to fully automatically acquire images at distinct positions at cytological specimens using a conventional computer controlled microscope and an autofocus algorithm. Based on the thus obtained images we automatically detect p16 ${ }^{i n k} 4 a_{-}$positive objects. This detection in turn is based on an analysis of the color distribution of the p16 ${ }^{i n k} 4 a$ marker in the Lab-colorspace. A Gaussian-mixture-model is used to describe this distribution and the method described in this paper so far achieves a sensitivity of up to $90 \%$.
\end{abstract}

Keywords: Methods: pre-processing, feature extraction, Modalities: optical imaging, microscopy imaging, Diagnostic Task: detection, diagnosis

\section{INTRODUCTION}

Conventional histopathological methods for cancer detection are based on tissue specimens obtained by a biopsy. The need for a biopsy for the diagnostic process renders these histopathological methods unacceptable for preventive diagnostics, viz, screening applications. Hence, these methods can only be applied in curative diagnostics, i.e., in cases where patients already exhibit symptoms that need treatment. Furthermore many of these biopsies are in retrospect unnecessary, e.g., in $95 \%$ of cases for thyroid nodules. ${ }^{1}$

Cytopathological investigations, in contrast, require only painlessly accessible smears or fine-needle-aspiration biopsies (FNABs). The former can be obtained from any accessible mucosa of the human body (e.g., oral or cervical mucosa) while the latter can be obtained from different organs (e.g., thyroid nodules or prostate). Depending on the cancer, cytopathological investigations can detect cancer up to three years ahead of histopathological methods $^{2}$ while, at the same time, unnecessary biopsies can be avoided, and specimens are obtained without stress for the patients.

The cytopathological methods are based on a microscopic analysis of cells. These cells have to be stained to observe any desirable feature under the microscope. Several stains are commonly applied to observe or measure different characteristics of the cells in question. One very important and most often used stain is Feulgen, which stains the DNA in the nucleus stoichiometrically and hence allows to measure the amount of DNA in the cell. A population of cancer cells shows a different distribution in the thus obtained DNA-histograms. Since

Further author information: (Send correspondence to André A. Bell)

André A. Bell: E-mail: andre.bell@lfb.rwth-aachen.de, Telephone: +49 (241) 8027860 


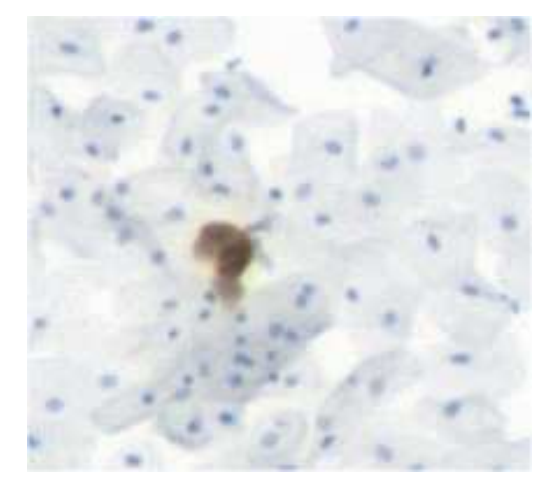

Figure 1. Image from a smear from a cervical brush biopsy. Nuclei (dark blue), cytoplasm (light blue), and immunocytochemically marked (positive) cells (brown) can be observed.

these investigations are quite time consuming, they have to be either automated or applied only to a subset of suspicious specimens. The latter strategy will be advantageous in the particular case of screening applications, since for the early discovery of cancer a dominating amount of specimens is expected to be negative. To perform a selection, however, into specimens to be retained for further analysis or to be discarded, we require a simple but highly sensitive preceeding analysis in order not to discard false-negatives. This can be achieved by means of so-called immunocytochemical markers. For many cancers a deregulation of the cell cycle can be observed, which is accompanied by an increased production of specific proteins within the cells. These in turn can be detected by a immunocytochemical markers, of which the p16 ${ }^{\text {ink } 4 a}$ marker is one example. Applying such a marker allows to remove the majority of cancer negative cells and specimens from the following DNA image cytometry (DNA-ICM), due to the markers' high sensitivity.

As an example we consider cervical cancer screening. To reach a high overall sensitivity and specificity, at first, for the high volume of specimens a p16 ${ }^{i n k} 4 a$ staining and fully automatic analysis is applied. Now, only the suspicious specimens need further analysis. The overall specificity can now be increased by application of the more time consuming DNA-ICM to the subset of suspicious specimens. This paper deals with the first step, the fully automatic analysis of $\mathrm{p} 16^{i n k 4 a}$ stained specimens.

The paper is organized as follows. In section 2 the equipment and the cytopathological material are summarized. This is followed by the description of the methods involved, i.e., the autofocus and slide screening in section 3.1 and the $16^{i n k 4 a}$ marker detection in section 3.2. Finally the experiments and results are presented in section 4 , followed by a summary and a discussion in section 5 .

\section{MATERIAL}

Cytopathological diagnostic methods are based on a microscopic analysis of the specimens. Our microscope workstation is composed of a 3-chip CCD RGB camera (CV-M90, JAI) mounted on top of a microscope (DMLA, Leica). The microscope is equipped with dry objectives (5x,10x, 20x, and 40x magnification) and oil immersion objectives (63x and 100x magnification). Furthermore, the microscope has a motorized xyz-stage for positioning and focusing of the slide. Camera and microscope are controlled by a PC for image capture. All images were acquired with the $5 \mathrm{x}$-objective lens $(\mathrm{NA}=0.15)$, resulting in a pixel edge length of $1.25 \mu m$. Seven specimens $\left(S_{1}-S_{7}\right)$ of cervical smears were stained with hematoxilin (blue) and, at the same time, with the p16 ink $4 a$ marker (red-brown) (Fig. 1). A further specimen $\left(S_{8}\right)$ was prepared, which has been stained with p16 ${ }^{i n k} 4 a$ only.

\section{METHODS}

The overall algorithm can be divided into two major parts, first, an autofocus in combination with systematic acquisition of images at every distinct position of the slide and, second, the analysis of each image obtained that way. 


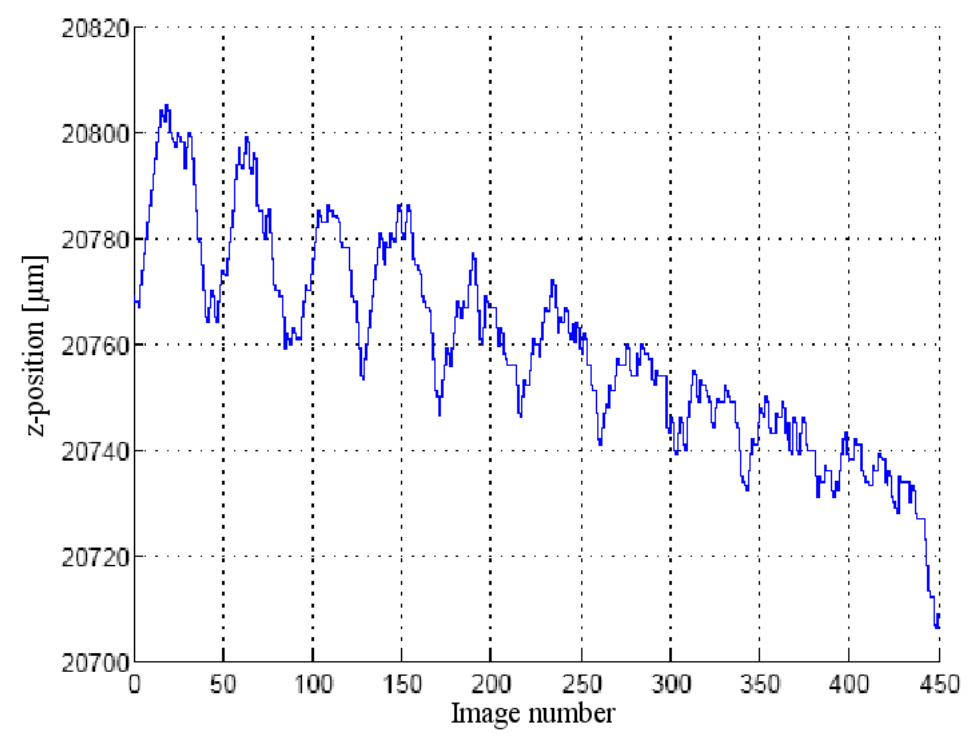

Figure 2. This diagram shows the focus position as a function of the xy-position, i.e., the image number. Starting from the top-left corner images have been acquired meander-like over the full slide until reaching the bottom-right corner. The overall trend represents the change of the focus position from top to bottom, while the wave form of the graph shows the change of the focus position from left to right and back.

\subsection{Autofocus and Image Acquisition}

Autofocus methods can be divided into active and passive methods. An active autofocus is based on the measurement of the distance to the object, e.g., by infrared or ultrasound, while a passive autofocus method is based on a focus score calculated from the acquired digital image itself. Hence, this focus score measures the sharpness of the image and defines the accuracy of the focus position. A variety of focus scores have been proposed, e.g., summing the magnitude of the gradient,${ }^{3}$ sum-modified-Laplacian, ${ }^{4}$ and sum-modulus-difference (SMD), ${ }^{5}$ and compared for microscopy. ${ }^{6,7}$

The SMD focus score was found to perform suitably, while at the same time it is computationally inexpensive. ${ }^{7}$ The calculation of the SMD obeys

$$
\mathrm{SMD}=\sum_{\Omega}|I(x, y)-I(x-1, y)|+|I(x, y)-I(x, y-1)|
$$

where $I(x, y) ;(x, y) \in \Omega$ represents the image intensity. The SMD-value is the sum of the absolute pixel value differences in $\mathrm{x}^{-}$- and $\mathrm{y}$-direction, over the image region $\Omega$. The calculated differences are a discrete approximation of the derivatives, which in turn correspond to a highpass-filtered version of the image. This highpass filter emphasizes high frequency content and is therefore sensitive to edges as well as noise.

Microscopy images of cytopathological specimens show cells over a mostly homogeneous background. Hence, the focus score can be made more robust against background noise by restricting the region $\Omega$ to regions of interest. Since we need to focus on nuclei, we extract the region of interest that should be focused on, viz the borders of the nuclei. Towards this end, we exclude the background using an Otsu-threshold ${ }^{8}$ on the luminance of the image. This mask is refined by closing holes. The final mask is calculated by subtracting an eroded (smaller) mask from a dilated (larger) one, giving a mask of the borders. Calculating the SMD-value within this region is robust against background noise.

In general we now will have to calculate the SMD focus score for each of several distinct possible focus positions. These can be acquired by driving the microscope stage up to an upper limit $z_{\max }$ and lowering the 
position in steps of $\Delta z$ down to position $z_{\text {min }}$. For each $\mathrm{z}^{-}$-position the focus score can calculated and the best focus score represents the optimal focus position. For accurate p16 ${ }^{\text {ink }} 4 a$ marker detection, we are interested in acquiring images of the full slide. This is achieved by driving the microscope stage meander-like from the top-left to the bottom-right corner of the slide (Fig. 2). As observed in Fig. 2, the focus position varies only slightly from position to position. Hence, to gain speed we have implemented our autofocus as a tracking autofocus.

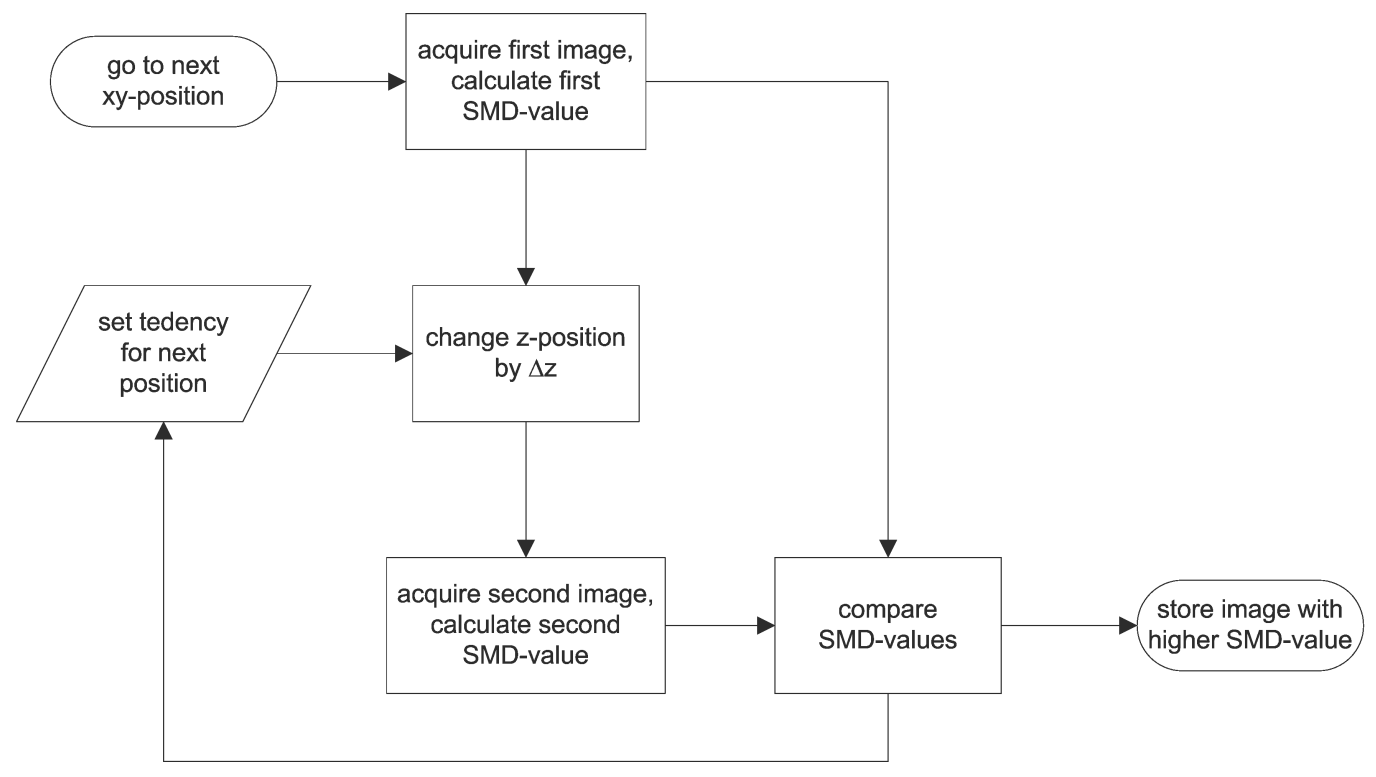

Figure 3. Flow chart for the tracking autofocus. The algorithm starts by driving the microscope stage to the next xy-position on the slide. A first image is acquired and the focus score is calculated. Depending on the tendency the z-position is adjusted by an amount of $\Delta z$, followed by the acquisition of a second image and calculating its corresponding focus score. The image with the highest focus score is stored for the current xy-position and the tendency is changed accordingly.

Consider the first position (top-left corner) to be accurately focused. We now assume that the focus position will change from one xy-position to its next neighbouring position by a small amount up or down, thus following a tendency. We drive the microscope stage to the next xy-position and acquire a first image $I_{1}$. Then we change the focus position by a specific amount $\Delta z$ along the current tendency up or down and acquire a second image $I_{2}$. For both images the focus score is calculated as described above, and the image with the superior sharpness is stored. Depending on the outcome, which image has the higher focus score, the tendency is adjusted (Fig. 3).

For this tracking autofocus algorithm an appropriate $\Delta z$ has to be chosen. According to Rayleigh's formula (2), the depth of field is given by

$$
\Delta z_{\mathrm{DOF}}=\frac{n \lambda}{2 \cdot \mathrm{NA}^{2}}
$$

with $n$ the refractive index of the medium (here air: $\mathrm{n}=1.0), \lambda$ the wavelength of the light, and NA the numerical aperture of the microscope objective ( $\mathrm{NA}=0.15$ for our $5 \mathrm{x}$ objective lens). For $\lambda=550 \mathrm{~nm}$ this then results in $\Delta z_{\mathrm{DOF}} \approx 12.22 \mu \mathrm{m}$. Hence, the depth of field $\Delta z_{\mathrm{DOF}}$ is an upper limit for the focus step width $\Delta z$. Nevertheless, $\Delta z$ cannot be chosen arbitrarily small, since it must be large enough to measure differences in sharpness despite noise induced variations of the focus score. Therefore, we have empirically evaluated the focus score over different $\mathrm{z}-$ positions. On $n=10$ distinct positions on a slide, we have manually adjusted the focus position $\left(z_{\text {ideal }}\right)$. Subsequently, the $\mathrm{z}$-position has been automatically changed from $z_{\text {ideal }}-50 \mu m$ to $z_{\text {ideal }}+50 \mu \mathrm{m}$ in steps of $\Delta z=1 \mu \mathrm{m}$. On each of these $\mathrm{z}$-positions 10 images have been acquired, resulting in 1000 images per xy-position (Fig. 4). 


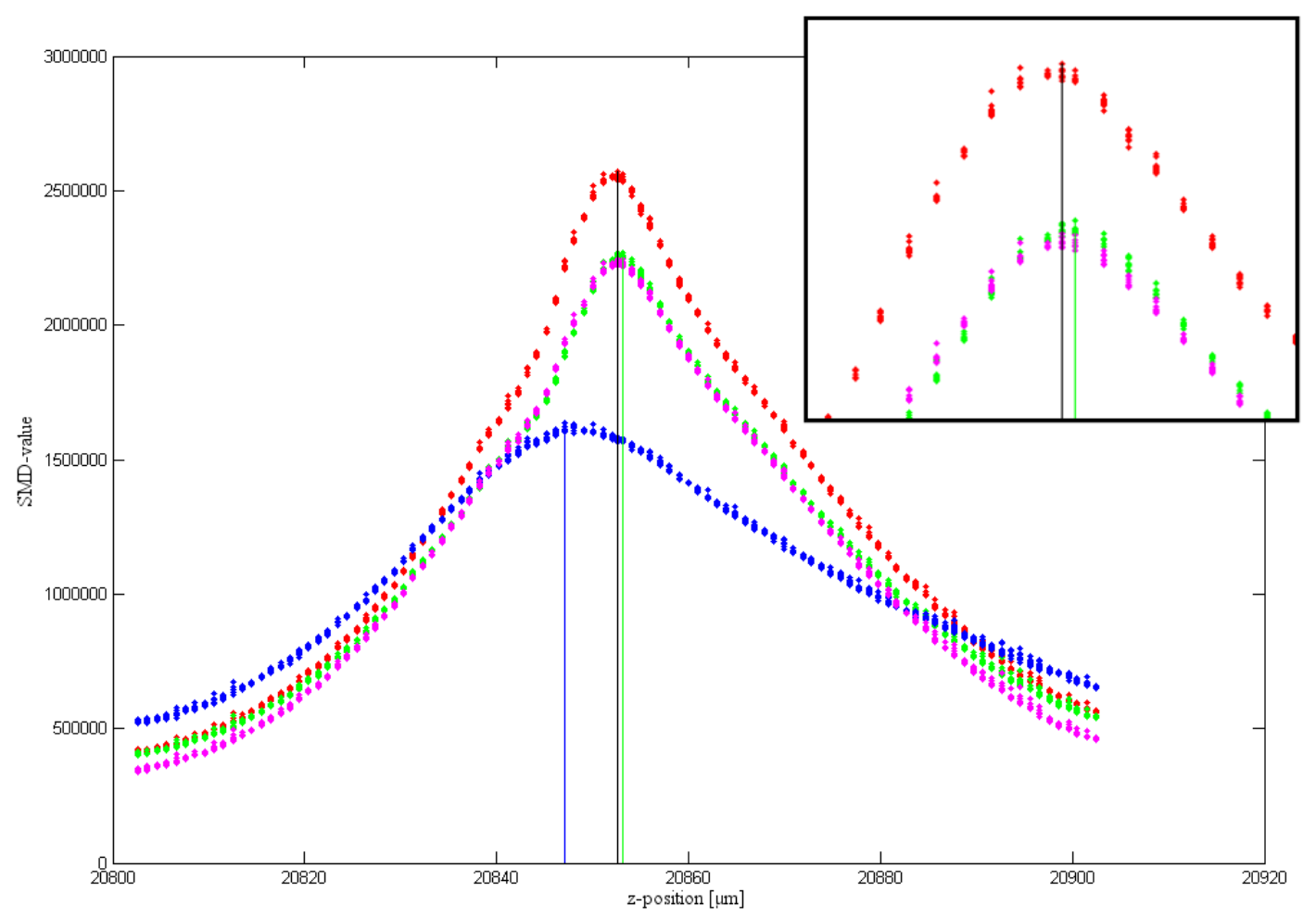

Figure 4. SMD value over different $z$-positions for red, green, blue, and luminance (magenta) channel. The vertical lines indicate the maximum SMD value for each channel (user selected optimum $z_{\text {ideal }}$ in black). Note, that luminance and red channel nearly perfectly match with the user selected best focus position. On each z-position 10 measurements have been obtained, showing the influence of noise on the focus score.

It has to be prevented, that, due to noise induced variations, a comparison between two focus scores results in the wrong tendency selection. As can be seen from Fig. 4 this can be achieved for $\Delta z=4 \mu m$. To verify this, we have acquired a complete slide with different values of $\Delta z \in\{2 \mu m, 4 \mu m, 6 \mu m\}$. For small steps, the tracking was in a few cases too slow for the amount of focus-position change. On the other hand, large steps resulted in some cases in imprecise autofocus results. The results of the autofocus for all full slide scans have been manually reviewed, and found to be satisfactory for the chosen stepwidth of $\Delta z=4 \mu m$.

\subsection{Marker detection}

After automatic acquisition of the full slide, the images have to be processed and p16 ink4a marker positive cells have to be detected. As described in section 2, all specimens are stained using a morphological stain, i.e., a stain that reveals nuclei and cytoplasm to allow a simultaneous analysis of the cell morphology (typically some kind of blue), alongside with the immunocytochemical marker p16 ink $4 a$, which has a specific different color expression (typically some kind of red-brown). All acquired images $I$ are corrected for shading and dark current according to

$$
I_{\text {corr }}(x, y)=\frac{I(x, y)-I_{\text {black }}(x, y)}{I_{\text {white }}(x, y)-I_{\text {black }}(x, y)}
$$

with $I_{\text {black }}$ and $I_{\text {white }}$ being the dark current and white reference image respectively. 


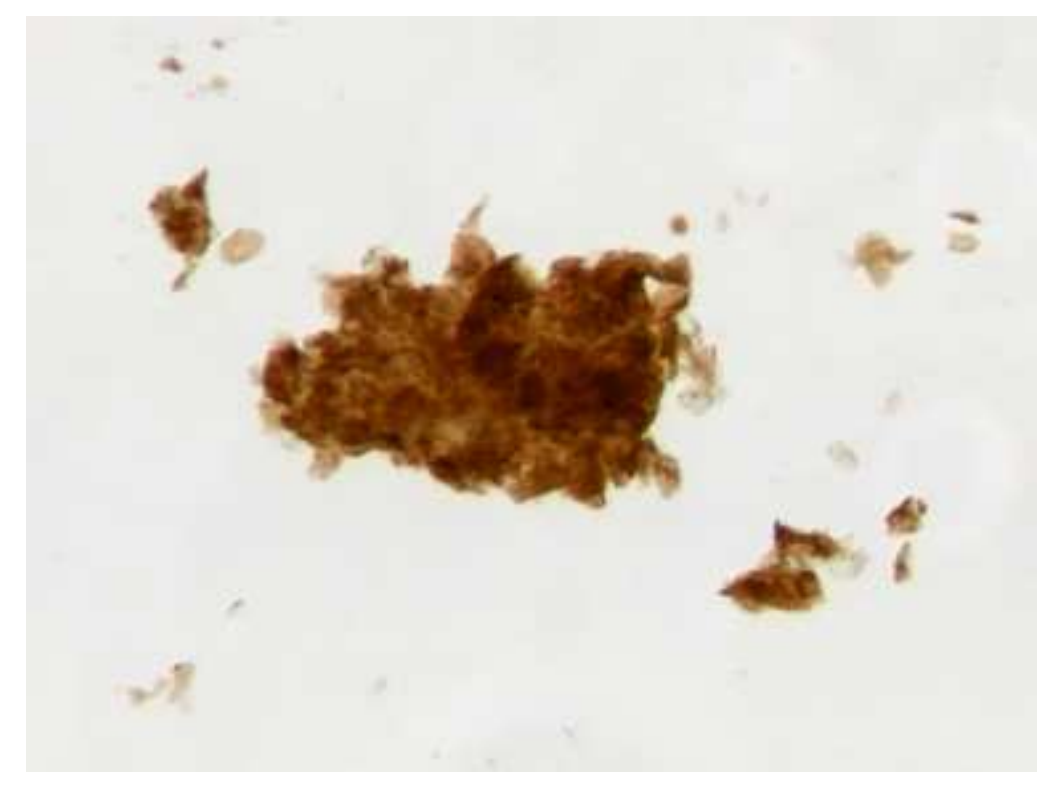

Figure 5. Image of cells that have been stained immunocytochemically only. Note that cells outside the region of marker positive cells are now invisible, due to the lack of the counterstain (Hematoxilin, blue).

The stain specific colors are represented best in the Lab-colorspace, where two distinct colors have a measureable distance proportional to the subjective difference of a human observer.

The detection algorithm aims at finding the p16 ${ }^{i n k} 4 a$ marker-specific color. Based on specimen $S_{8}$, which is stained with p16 ink $4 a$ only, a training set of images has been acquired (Fig. 5). Due to deficiencies in the imaging system, such as chromatic aberrations, misalignment of the three CCD sensors, or blooming effects, the images are always compromised by different additional colors. Hence, the peaks in a histogram (Fig. 6) over the ab-plane in the Lab-colorspace are affected by noise, caused by these acquisition imperfections.
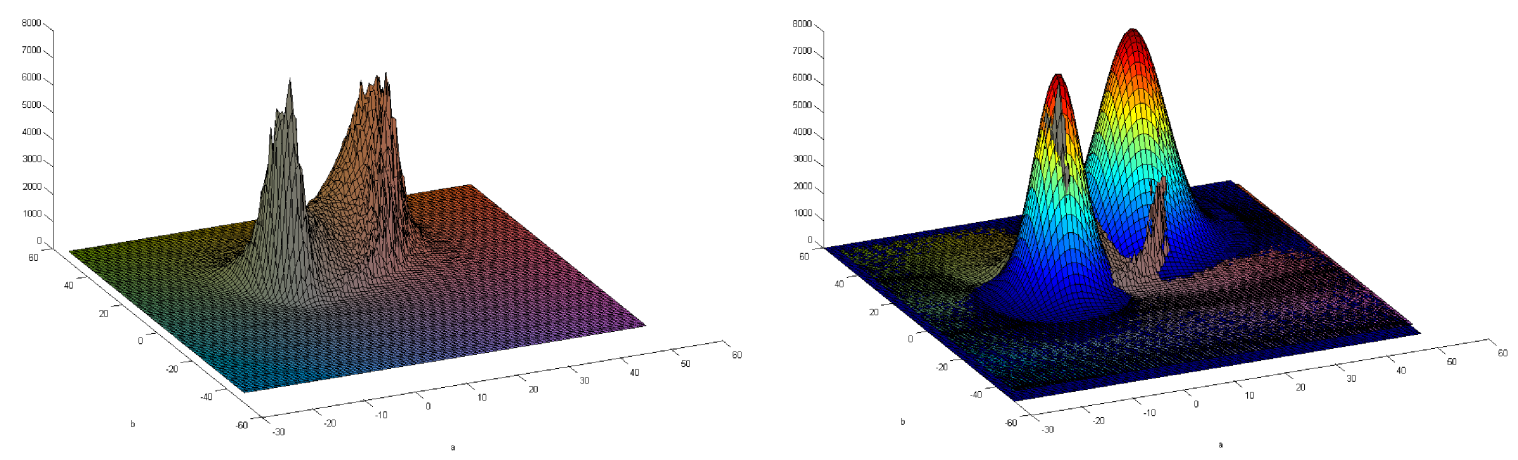

Figure 6. Histogram of the color values in the training data over the ab-plane in the Lab-colorspace. Note the mostly bi-modal distribution of the colors of the marker (red-brown) and background (white-gray) in the left plot. On the right, the same plot together with the fitted Gaussian-mixture-model (4). 
Fitting a Gaussian-mixture-model

$$
p(\mathbf{x})=\xi_{1} p_{1}(\mathbf{x})+\xi_{2} p_{2}(\mathbf{x}) ; \sum_{i} \xi_{i}=1
$$

with

$$
p_{i}(\mathbf{x})=\frac{1}{(2 \pi)^{N / 2}\left|\boldsymbol{\Sigma}_{\mathbf{i}}\right|^{1 / 2}} e^{-\frac{1}{2} \tilde{\mathbf{x}}^{T} \boldsymbol{\Sigma}_{\mathbf{i}}^{-1} \tilde{\mathbf{x}}} ; \tilde{\mathbf{x}}=\mathbf{x}-\overline{\mathbf{x}} .
$$

to this distribution allows to describe the distribution of the p16 $6^{\text {ink }} 4 a$ color despite the presence of erroneous colors. Since we are interested in detecting p16ink $4 a$ marker positive cells, we can now select that Gaussian function from the Gaussian-mixture-model, which describes the distribution of the p16 ${ }^{\text {ink }} 4 a$ color. Transferring the confidence interval of this Gaussian function back to the RGB-colorspace of the camera, a subspace is identified along with a probability of each RGB-value to be p16 $6^{i n k} 4 a$ positive. After such a teach-in of the algorithm the resulting data can now be applied to every image acquired from other slides.

Hence, for every pixel in each image thus obtain a probability whether or not it is marker positive. Based on a threshold we select pixels of minimum probability. Neighboring pixels are now grouped into objects and those objects which are too small to be a cell are removed. For each object the mean pixel probability is computed

$$
p_{\text {Object }}=\frac{1}{N} \sum_{N} p_{\text {pixel }}
$$

Based on this object-probability $p_{\text {Object }}$ the classification of $\mathrm{p} 16^{i n k 4 a}$ positive objects can now be based on a threshold (Fig. 7).

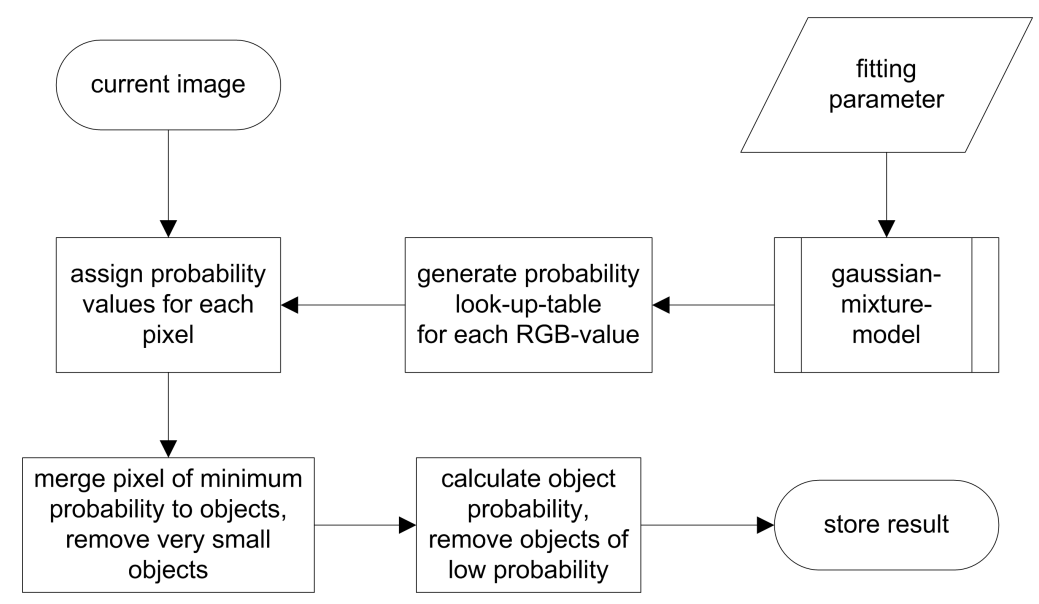

Figure 7. Flow chart of the overall detection algorithm. For each pixel the marker positive probability is chosen from a precomputed lookup-table. This lookup-table in turn is based on the Gaussian-mixture-model, which has been fitted to the trainingsdata.

\section{EXPERIMENTS AND RESULTS}

We have applied the algorithm to our sample set of cytopathological specimens $S_{1}-S_{7}$. The autofocus acquires images of satifactory quality on all specimens. To evaluate the results of the p16 ink $4 a$ marker detection we divided these sets into different categories, which reflect the differences in intensity of the morphological stain. Depending on this intensity, the contrast between the morphological and the immunocytochemical stain p16 ${ }^{\text {ink } 4 a}$ 
ranges from low to high. The detection rate, i.e., the sensitivity (7) of the algorithm, is directly related to this contrast,

$$
\text { sensitivity }=\frac{\mathrm{TP}}{\mathrm{TP}+\mathrm{FN}}
$$

where TP and FN are true positive and false negative respectively (table 1). These values have been determined with respect to manual review of the acquired images with respect to p16 ${ }^{i n k} 4 a$ positive cells. Lower intensity of the morphological stain, and hence in turn higher contrast between the stains, leads to better results of up to $90 \%$ sensitivity. For an intensive morphological stain the light absorption of the cells increases and the contrast to the color of p16 ink4a drops, which leads to a difficult separability. Only a few RGB-values remain for the decision and the sensitivity drops to $40 \%$ on these specimens. The results are summarized in table 1 and examples of acquired images and corresponding marker detection are shown in figures 8-14.

\begin{tabular}{|c|c|c|c|c|c|c|c|c|c|}
\hline specimen & contrast & P & N & TP & FP & TN & FN & sensitivity & specificity \\
\hline \hline$S_{7}$ & very low & $6.8 \%$ & $93.2 \%$ & $2.8 \%$ & $4 \%$ & $88.6 \%$ & $4.6 \%$ & $41 \%$ & $95 \%$ \\
\hline$S_{4}$ & low & $21.8 \%$ & $78.2 \%$ & $12.5 \%$ & $9.3 \%$ & $66.7 \%$ & $11.5 \%$ & $57 \%$ & $85 \%$ \\
$S_{1}$ & low & $20 \%$ & $80 \%$ & $13.3 \%$ & $6.7 \%$ & $67 \%$ & $13 \%$ & $67 \%$ & $84 \%$ \\
$S_{5}$ & low & $43.2 \%$ & $56.8 \%$ & $31 \%$ & $12.2 \%$ & $47.8 \%$ & $9 \%$ & $72 \%$ & $84 \%$ \\
\hline$S_{2}$ & normal & $66.6 \%$ & $33.4 \%$ & $55.8 \%$ & $10.8 \%$ & $24 \%$ & $9.4 \%$ & $84 \%$ & $72 \%$ \\
\hline$S_{3}$ & very good & $57.3 \%$ & $42.7 \%$ & $51.5 \%$ & $5.8 \%$ & $31.7 \%$ & $11 \%$ & $90 \%$ & $74 \%$ \\
$S_{6}$ & very good & $14.4 \%$ & $85.6 \%$ & $13.1 \%$ & $1.3 \%$ & $76.1 \%$ & $9.5 \%$ & $91 \%$ & $89 \%$ \\
\hline
\end{tabular}

Table 1. Results for the detection of marker positive images. The first column shows the specimen number, the second the contrast between counterstain and immunocytochemical marker stain. Columns $\mathrm{P}$ and $\mathrm{N}$ show the amount of marker positive and marker negative images, as obtained from a manual review. In the following four columns the true positive (TP), false positive (FP), true negative $(\mathrm{TN})$, and false negative (FN) results of the automatic marker detection are shown. This is complemented with the sensitivity and specificity of the detection algorithm in the last two columns. Note, that the sensitivity of the test increases with the contrast between counterstain and immunocytochemical marker. For very good contrast, i.e., an unobtrusive counterstain, as is the case for $S_{3}$ and $S_{6}$, the detection so far achieves best results.
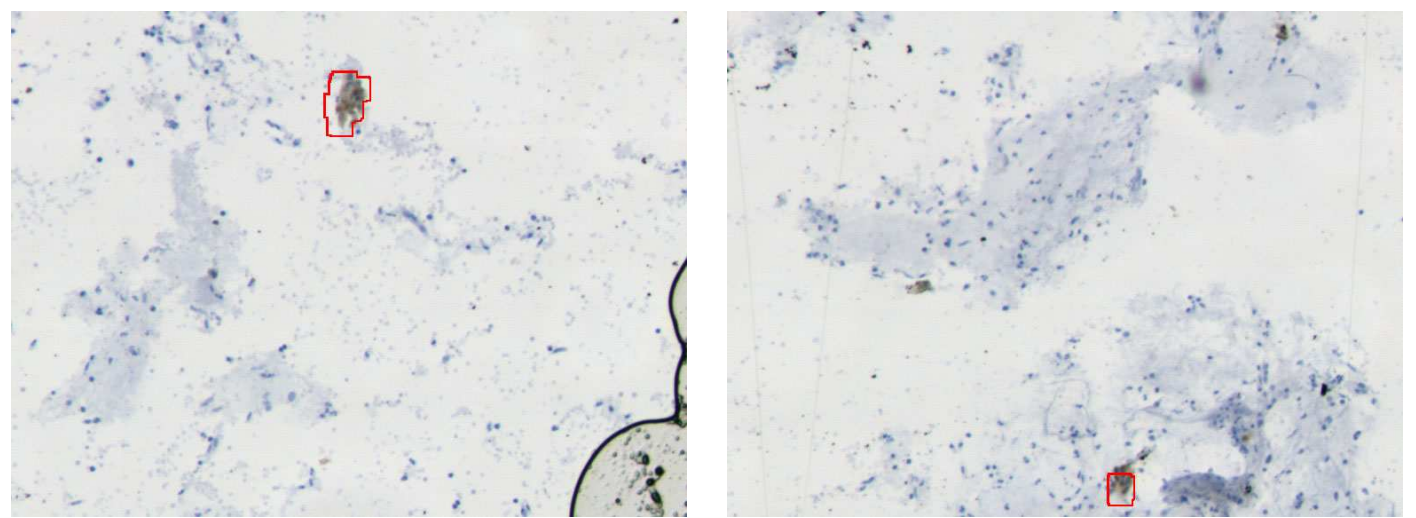

Figure 8. Samples from specimen $S_{1}$. Note the low contrast between the two colors in the detected areas.

\section{SUMMARY AND DISCUSSION}

The autofocus is adaptive to the material under scrutiny, viz, the cells. Based on an analysis of the focus positions over an entire slide we demonstrated the feasibility of a tracking autofocus for this material in a microscopy environment. After fully automatic acquisition of the images an analysis of marker positivity follows. 

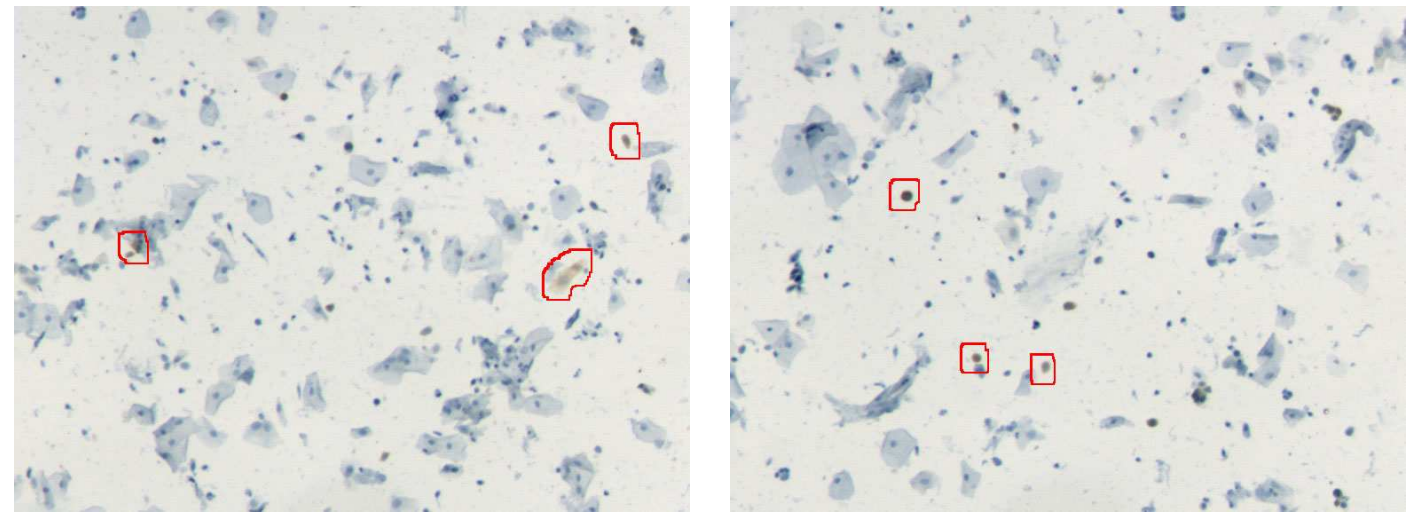

Figure 9. Samples from specimen $S_{2}$. The overall contrast rating is normal.
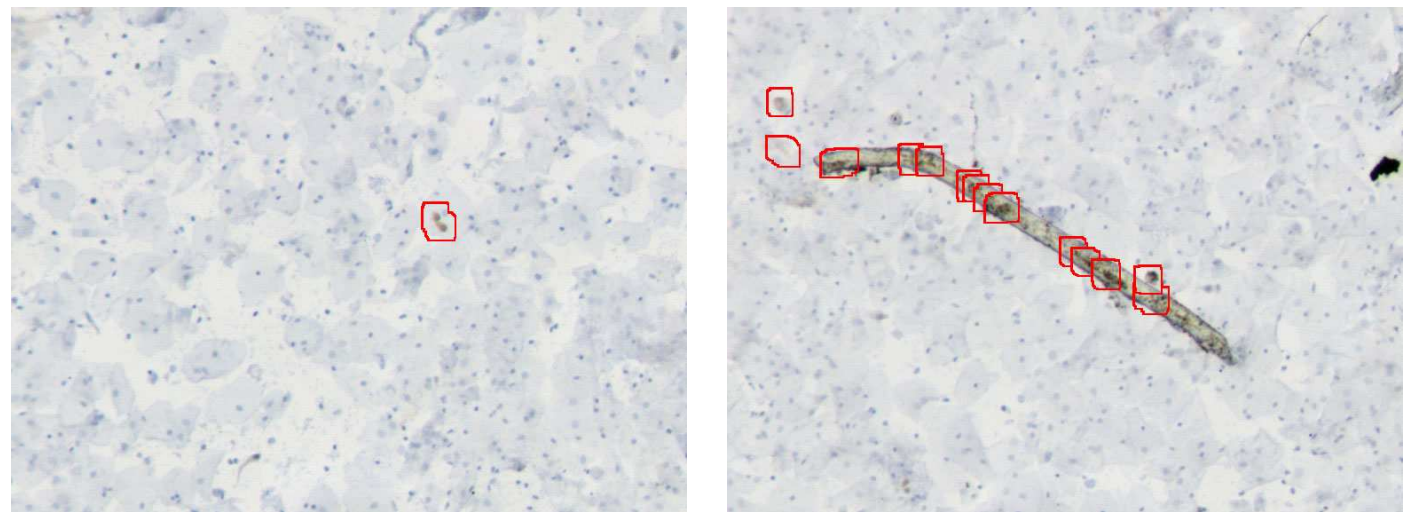

Figure 10. Samples from specimen $S_{3}$. The overall contrast between the two colors is very good, i.e., there is only a light counterstain. Note the false positive detections in the right image, which are caused by an artifact.

This is based on a teach-in from a specimen, which has only been stained with p16 ink $4 a$ and hence, is well suited to measure the properties of this specific color. The color-distribution of this specimen was modelled using a Gaussian-mixture-model fitted into the histogram over the ab-plane in the Lab-colorspace. Based on the parameters of this model an object probability for objects in the image is calculated. Marker positivity for these objects is computed by a straight forward threshold operation.

The tracking autofocus works and reliably is suitable for other, similar applications. Nevertheless, there are two tradeoffs. First, there must be enough cells, i.e., areas without observable material will cause this autofocus to fail. Second, since the cells are only observable after a staining process, all cells have to be stained with a counterstain. Based on the images acquired so far, the detection of the immunocytochemical marker directly depends on the intensity of the counterstain. If this intensity is too strong, the contrast between the colors of counterstain and p16 ink $4 a$ marker decreases and hence, the marker detection fails. From this it follows, that best detection results can be achieved for an unobtrusive counterstain, which can be achieved by an appropriate staining protocol. An manual analyis of the detection results showed that false positives detections are nearly allways borders of artifacts (dust particles, air bubbles, etc.), while p16 ${ }^{\text {ink } 4 a}$ positive cells, which have not been detected (false negatives) are always due to low contrast between the two stains. Based on the results achieved so far, we will investigate further detection algorithms in the future, e.g., the refinement of the algorithm with respect to the artifacts, which cause false positives. Furthermore, we will apply the algorithm to different other immunocytochemical markers. 

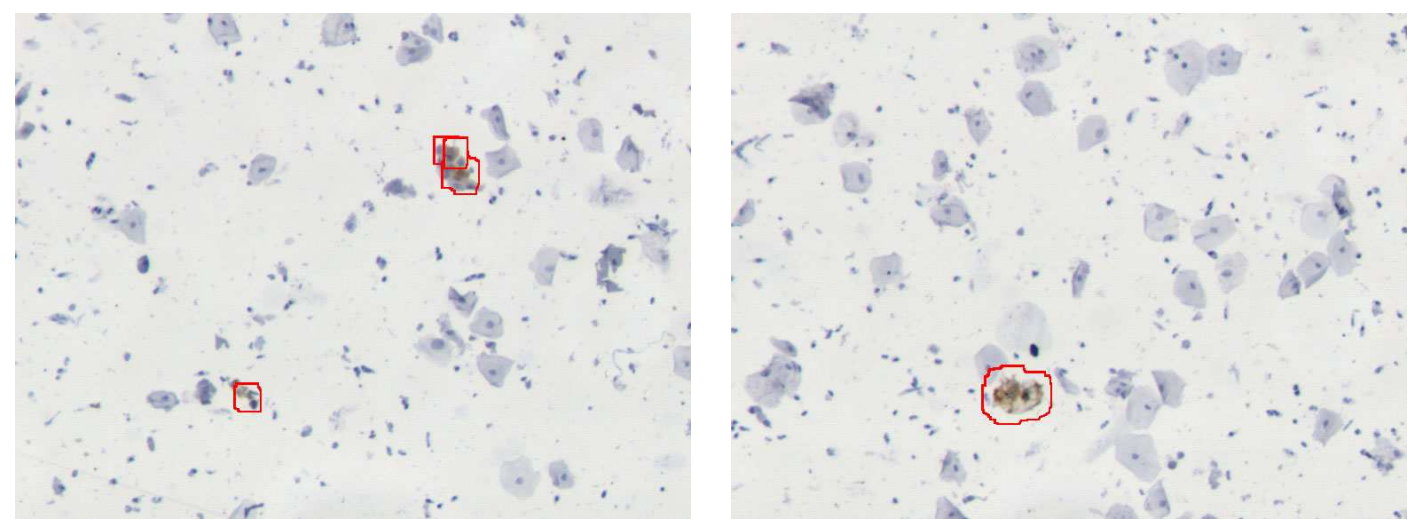

Figure 11. Samples from specimen $S_{4}$. The overall contrast is rated low.
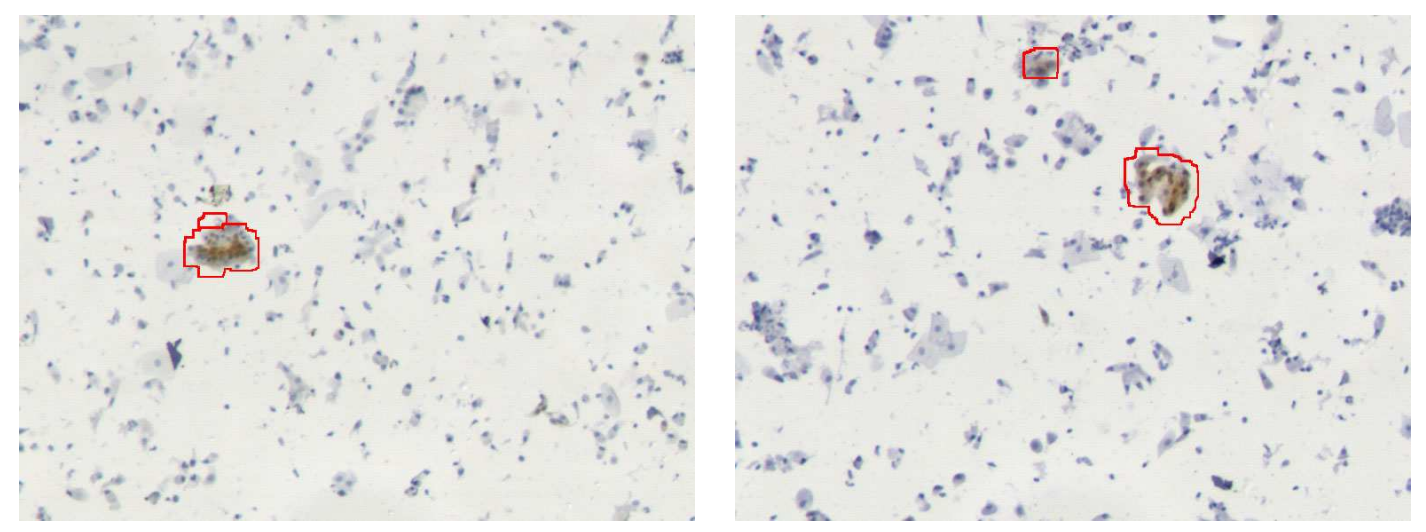

Figure 12. Samples from specimen $S_{5}$. The overall contrast rating is low.
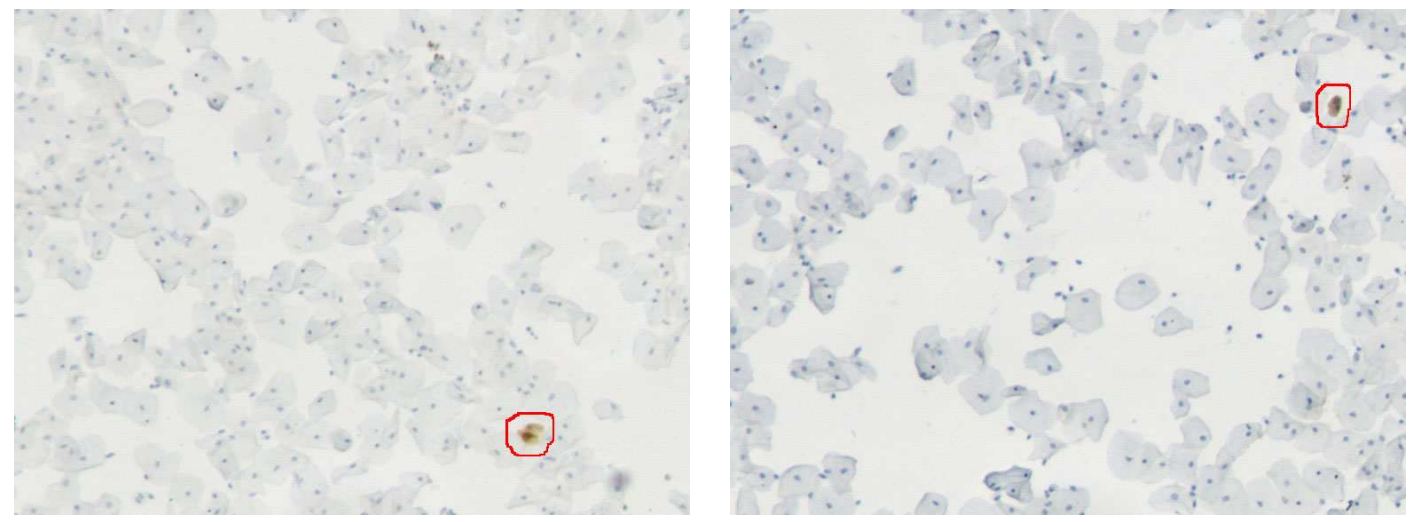

Figure 13. Samples from specimen $S_{6}$. There is only a very light counterstain and thus a very good contrast between counterstain and immunocytochemical marker.

\section{ACKNOWLEDGMENTS}

This work is supported by the Viktor-and-Mirka-Pollak-Fund for Biomedical Engineering. 

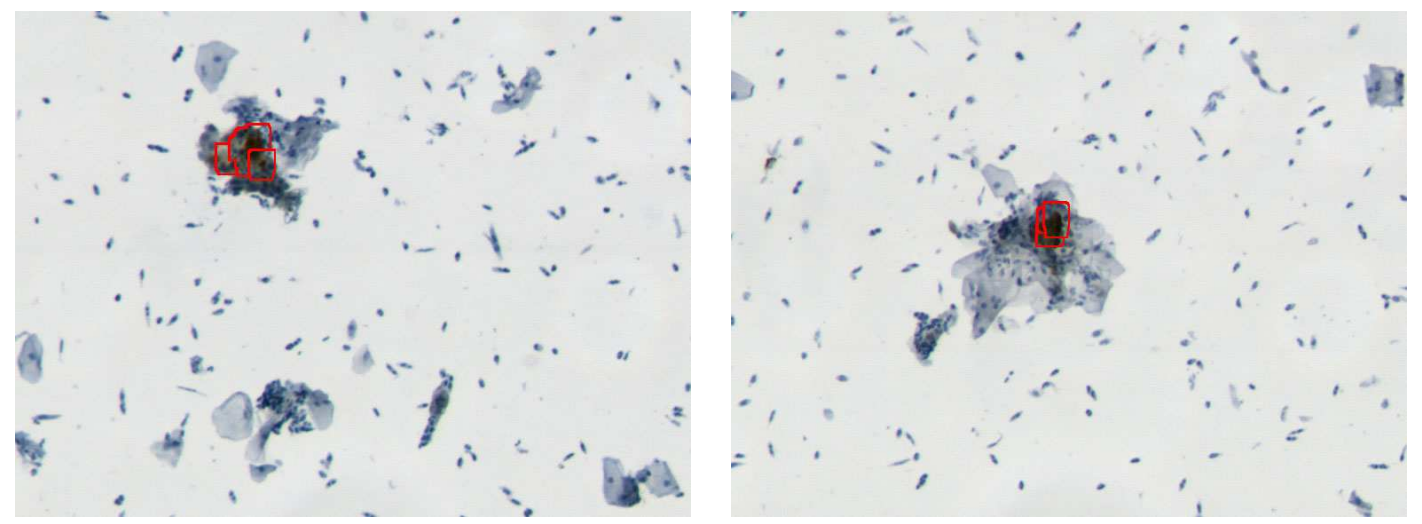

Figure 14. Samples from specimen $S_{7}$. The contrast and hence the detection rate drops, due to the intensive counterstain.

\section{REFERENCES}

1. A. Carpi, E. Ferrari, M. Toni, A. Agripanti, A. Nicolini, and G. D. Coscio, "Needle aspiration techniques in preoperative selection of patients with thyroid nodules: a long-term study.," J Clin Oncol 14(5), pp. 1704$1712,1996$.

2. T. Remmerbach, H. Weidenbach, N. Pomjanski, K. Knops, S. Mathes, A. Hemprich, and A. Böcking, "Cytologic and DNA-cytometric early diagnosis of oral cancer," Anal Cell Pathol 22(4), pp. 211-221, 2001.

3. J. M. Tenenbaum, Accomodation in computer vision. PhD thesis, Stanford University, 1970.

4. S. K. Nayar and Y. Nakagawa, "Shape fom focus," IEEE Transactions on Pattern Analysis and Machine Intelligence 16(8), pp. 824-831, 1994.

5. R. Jarvis, "Focus optimization criteria for computer image processing," Microscope 24(2), pp. 163-180, 1976.

6. L. Firestone, K. Cook, K. Culp, N. Talsania, and K. Preston, "Comparison of Autofocus Methods for Automated Microscopy," Cytometry 12, pp. 195-206, 1991.

7. C. Kang-Sun, L. Jun-Suk, and K. Sung-Jae, "New autofocusing technique using the frequency selective weighting median filter for video cameras," IEEE Transactions on Consumer Electronics 45(3), pp. 820-827, 1999.

8. N. Otsu, "A threshold selection method from gray level histograms," IEEE Transactions on Systems, Man and Cybernetics 9, pp. 62-66, 1979. 